\title{
EXAMPLES OF RIGID AND FLEXIBLE SEIFERT FIBRED CONE-MANIFOLDS
}

\author{
ALEXANDER KOLPAKOV* \\ Department of Mathematics, University of Fribourg, Ch. du Musée, 23, CH-1700 Fribourg, Switzerland \\ e-mail: kolpakov.alexander@gmail.com
}

(Received 8 November 2011; revised 15 June 2012; accepted 30 August 2012; first published online 25 February 2013)

\begin{abstract}
The present paper gives an example of a rigid spherical cone-manifold and that of a flexible one, which are both Seifert fibred.

2010 Mathematics Subject Classification. 53A35, 57R18, 57M25.

1. Introduction. The theory of three-dimensional orbifolds and cone-manifolds attracts attention of many mathematicians since the original work of Thurston [29]. An introduction to the theory of orbifolds could be found in [29, chapter 13]. For a basic introduction to the geometry of three-dimensional cone-manifolds and cone-surfaces, we refer the reader to [6]. The main motivation for studying three-dimensional conemanifolds comes from Thurston's approach to geometrisation of three-orbifolds: threedimensional cone-manifolds provide a way to deform geometric orbifold structures. The orbifold theorem has been proven in full generality by M. Boileau, B. Leeb and J. Porti (see [1, 2]).
\end{abstract}

One of the main questions in the theory of three-dimensional cone-manifolds is the rigidity problem. First, the rigidity property was discovered for hyperbolic manifolds (so-called Mostow-Prasad rigidity, see [19, 24]). After that, the global rigidity property for hyperbolic three-dimensional cone-manifolds with singular locus a link and cone angles less than $\pi$ was proven by S. Kojima [16]. The key result that implies global rigidity is due to Hodgson and Kerckhoff [13], who showed the local rigidity of hyperbolic cone manifolds with singularity of link or knot type and cone angles less than $2 \pi$. The de Rham rigidity for spherical orbifolds was established in $[\mathbf{2 6}, \mathbf{2 7}$. Detailed analysis of the rigidity property for three-dimensional cone-manifolds was carried out in $[31,32]$ for hyperbolic and spherical cone-manifolds with singularity a trivalent graph and cone angles less than $\pi$.

Recently, the local rigidity for hyperbolic cone-manifolds with cone angles less than $2 \pi$ was proven in $[\mathbf{1 8}, \mathbf{3 3}]$. However, examples of infinitesimally flexible hyperbolic conemanifolds had already been given in [5]. For other examples of flexible cone-manifolds, one may refer to $[\mathbf{1 5}, \mathbf{2 1}, \mathbf{2 8}]$.

The theorem of [32] concerning the global rigidity for spherical three-dimensional cone-manifolds was proven under the condition of being not Seifert fibred. Recall that due to [22], a cone-manifold is Seifert fibred if its underlying space carries a Seifert fibration such that components of the singular stratum are leafs of the fibration. In particular, if its singular stratum is represented by a link, then the complement is a Seifert fibred three-manifold. All Seifert fibred link complements in the three-sphere

*Supported by the Schweizerischer Nationalfonds SNF no. 200020-121506/1 and no. 200021-131967/1 
are described by [4]. In the present paper, we give an explicit example of a rigid spherical cone-manifold and a flexible one, which are both Seifert fibred. The singular locus for each of these cone-manifolds is a link and the underlying space is the three-sphere $\mathbb{S}^{3}$. The rigid cone-manifold given in the paper has cone-angles of both kinds, less or greater than $\pi$. The flexible one has cone-angles strictly greater than $\pi$. Deformation of its geometric structure comes essentially from those of the base cone-surface. However, hyperbolic orbifolds, which are Seifert fibred over a disc, are rigid. Their geometric structure degenerates to the minimal-perimeter hyperbolic polygon, as shown in [23]. These are uniquely determined by cone angles.

The paper is organised as follows: first, we recall some common facts concerning spherical geometry. In the second section, the geometry of the Hopf fibration is considered and a number of lemmas are proven. After that, we construct two explicit examples of Seifert fibred cone-manifolds. The first one is a globally rigid conemanifold and its moduli space is parameterised by its cone angles only. The second one is a flexible Seifert fibred cone-manifold. This means that we can deform its metric while keeping its cone angles fixed. Rigorously speaking, the following assertion is proven: the given cone-manifold has a one-parameter family of distinct spherical cone metrics with the same cone angles.

2. Spherical geometry. Below we present several common facts concerning spherical geometry in dimension two and three.

Let us identify a point $p=(w, x, y, z)$ of the three-dimensional sphere

$$
\mathbb{S}^{3}=\left\{(w, x, y, z) \in \mathbb{R}^{4} \mid w^{2}+x^{2}+y^{2}+z^{2}=1\right\}
$$

with an $\mathrm{SU}_{2}(\mathbb{C})$ matrix of the form

$$
P=\left(\begin{array}{cc}
w+i x & y+i z \\
-y+i z & w-i x
\end{array}\right)
$$

Then, replace the group Isom $\mathbb{S}^{3} \cong \mathrm{SO}_{4}(\mathbb{R})$ of orientation preserving isometries with its two-fold covering $S U_{2}(\mathbb{C}) \times S U_{2}(\mathbb{C})$. Finally, define the action of $\langle A, B\rangle \in$ $S U_{2}(\mathbb{C}) \times S U_{2}(\mathbb{C})$ on $P \in S U_{2}(\mathbb{C})$ by

$$
\langle A, B\rangle: P \longmapsto A^{t} P \bar{B} .
$$

Thus, we define the action of $S O_{4}(\mathbb{R}) \cong S U_{2}(\mathbb{C}) \times S U_{2}(\mathbb{C}) /\{ \pm$ id $\}$ on the threesphere $\mathbb{S}^{3}$.

By assuming $w=0$, we obtain the two-dimensional sphere

$$
\mathbb{S}^{2}=\left\{(x, y, z) \in \mathbb{R}^{3} \mid x^{2}+y^{2}+z^{2}=1\right\} .
$$

Let us identify a point $(x, y, z)$ of $\mathbb{S}^{2}$ with the matrix

$$
Q=\left(\begin{array}{cc}
i x & y+i z \\
-y+i z & -i x
\end{array}\right)
$$

which represents a pure imaginary unit quaternion $Q \in \mathbf{H}$. 
Instead of Isom ${ }^{+} \mathbb{S}^{2} \cong S O_{3}(\mathbb{R})$, we use its two-fold covering $S U_{2}(\mathbb{C})$ acting by

$$
A: q \longmapsto A^{t} q \bar{A}
$$

for every $A \in S U_{2}(\mathbb{C})$ and every $q \in \mathbb{S}^{2}$.

Equip each $\mathbb{S}^{3}$ and $\mathbb{S}^{2}$ with an intrinsic metric of constant sectional curvature +1 . We call the distance between two points $P$ and $Q$ of $\mathbb{S}^{n}(n=2,3)$ a real number $d(P, Q)$ uniquely defined by the conditions

$$
\begin{array}{r}
0 \leq d(P, Q) \leq \pi, \\
\cos d(P, Q)=\frac{1}{2} \operatorname{tr} P^{t} \bar{Q} .
\end{array}
$$

The next step is to describe spherical geodesic lines in $\mathbb{S}^{n}$. Let us recall the following theorem [25, Theorem 2.1.5].

THEOREM 1. A function $\lambda: \mathbb{R} \rightarrow \mathbb{S}^{n}$ is a geodesic line if and only if there are orthogonal vectors $x, y$ in $\mathbb{S}^{n}$ such that

$$
\lambda(t)=(\cos t) x+(\sin t) y
$$

Taking into account the preceding discussion, we may reformulate the statement above.

LEMMA 1. Every geodesic line (a great circle) in $\mathbb{S}^{3}$ (respectively, $\mathbb{S}^{2}$ ) could be represented in the form

$$
C(t)=P \cos t+Q \sin t
$$

where $P, Q \in S U_{2}(\mathbb{C})$ (respectively $P, Q \in \mathbf{H}$ ) satisfy orthogonality condition

$$
\cos d(P, Q)=0
$$

By virtue of this lemma, one may regard $P$ as the starting point of the curve $C(t)$ and $Q$ as the velocity vector at $P$, since $C(0)=P, \dot{C}(0)=\left.\frac{d}{d t} C(t)\right|_{t=0}=Q$ and $d(C(0), \dot{C}(0))=\frac{\pi}{2}$ (the latter holds up to a change of the parameter sign).

Given two geodesic lines $C_{1}(t)$ and $C_{2}(t)$, define their common perpendicular $C_{12}(t)$ as a geodesic line such that there exist $0 \leq t_{1}, t_{2} \leq 2 \pi, 0 \leq \delta \leq \pi$ with the following properties:

$$
\begin{gathered}
C_{12}(0)=C_{1}\left(t_{1}\right), C_{12}(\delta)=C_{2}\left(t_{2}\right), \\
d\left(\dot{C}_{12}(0), \dot{C}_{1}\left(t_{1}\right)\right)=d\left(\dot{C}_{12}(\delta), \dot{C}_{2}\left(t_{2}\right)\right)=\frac{\pi}{2} .
\end{gathered}
$$

We call $\delta$ the distance between the geodesics $C_{1}(t)$ and $C_{2}(t)$. Note, that for an arbitrary pair of geodesics their common perpendicular should not be unique.

For an additional explanation of spherical geometry, we refer the reader to [25] and [31, chapter 6.4.2]. 
3. Links arising from the Hopf fibration. The present section is devoted to the construction of a family of links $\mathcal{H}_{n}(n \geq 2)$, which we shall use later. These links have a nice property - each of them is formed by $n \geq 2$ fibres of the Hopf fibration. Recall that the Hopf map $h: \mathbb{S}^{3} \stackrel{\mathbb{S}^{1}}{\rightarrow} \mathbb{S}^{2}$ has geometric nature [14, p. 654]. Our aim is to prove a number of lemmas concerning the geometry of the Hopf fibration in more detail.

3.1. Links $\mathcal{H}_{n}$ as fibres of the Hopf fibration. The Hopf map $h$ is defined as follows [14]: for every point $(w, x, y, z) \in \mathbb{S}^{3}$ let its image on $\mathbb{S}^{2}$ be

$$
h(w, x, y, z)=\left(2(x z+w y), 2(y z-w x), 1-2\left(x^{2}+y^{2}\right)\right) .
$$

The fibre $h^{-1}(a, b, c)$ over the point $(a, b, c) \in \mathbb{S}^{2}$ is a geodesic line in $\mathbb{S}^{3}$ of the form

$$
C(t)=\frac{1}{\sqrt{2(1+c)}}((1+c,-b, a, 0) \cos t+(0, a, b, 1+c) \sin t) .
$$

The exceptional point $(0,0,-1)$ has the fibre $(0, \cos t,-\sin t, 0)$.

The line $C(t)$ is a great circle of $\mathbb{S}^{3}$ and can be rewritten in the matrix form

$$
C(t)=P(a, b, c) \cos t+Q(a, b, c) \sin t,
$$

where

$$
\begin{gathered}
P(a, b, c)=\frac{1}{\sqrt{2(1+c)}}\left(\begin{array}{cc}
(1+c)-i b & a \\
-a & (1+c)+i b
\end{array}\right), \\
Q(a, b, c)=P(a, b, c)\left(\begin{array}{ll}
0 & i \\
i & 0
\end{array}\right) .
\end{gathered}
$$

We call

$$
F(t)=\left(\begin{array}{ll}
1 & 0 \\
0 & 1
\end{array}\right) \cos t+\left(\begin{array}{cc}
0 & i \\
i & 0
\end{array}\right) \sin t
$$

the generic fibre $h^{-1}(0,0,1)$. Moreover, every fibre $h^{-1}(a, b, c)$ can be described as a circle $C(t)=P(a, b, c) F(t)$. Note, that $P(a, b, c)$ is an $S U_{2}(\mathbb{C})$ matrix. Thus $C(t)$ could be obtained from $F(t)$ by means of the isometry $\left\langle P(a, b, c)^{t}\right.$, id $\rangle$. For the exceptional point $(0,0,-1) \in \mathbb{S}^{2}$, we set

$$
P(0,0,-1)=\left(\begin{array}{cc}
0 & 1 \\
-1 & 0
\end{array}\right)
$$

It is known that every pair of distinct fibres of the Hopf fibration represents simply linked circles in $\mathbb{S}^{3}$ (the Hopf link). Thus, $n$ fibres form a link $\mathcal{H}_{n}$ whose every two components form the Hopf link. One can obtain it by drawing $n$ straight vertical lines on a cylinder and identifying its ends by a rotation through the angle of $2 \pi$. Hence, $\mathcal{H}_{n}$ is an $(n, n)$ torus link.

Another remark is that the $\mathcal{H}_{n}$ link could be arranged around a point in order to reveal its $n$th order symmetry, as depicted in Figure 1. This fact allows us to consider $n$-fold branched coverings of the corresponding cone-manifolds with singular locus $\mathcal{H}_{n}$ that appear in Section 4. 


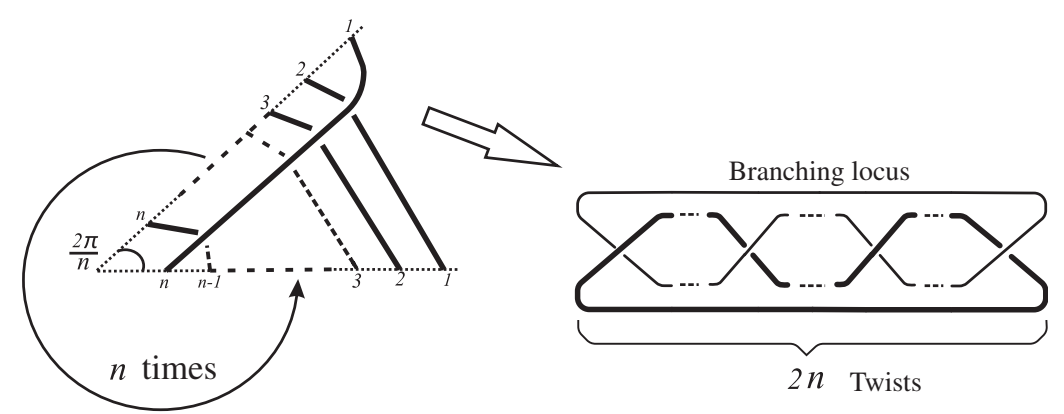

Figure 1. $n$-fold branched covering of $(2,2 n)$ torus link by $\mathcal{H}_{n}$.

3.2. Geometry of the Hopf fibration. Here and below, we use the polar coordinate system $(\psi, \theta)$ on $\mathbb{S}^{2}$ instead of the Cartesian one. Suppose

$$
\begin{gathered}
a=\cos \psi \sin \theta, b=\sin \psi \sin \theta, c=\cos \theta \\
0 \leq \psi \leq 2 \pi, \quad 0 \leq \theta \leq \pi
\end{gathered}
$$

and let

$$
M(\psi, \theta)=P(a, b, c)=\left(\begin{array}{cc}
\cos \frac{\theta}{2}-i \sin \psi \sin \frac{\theta}{2} & \cos \psi \sin \frac{\theta}{2} \\
-\cos \psi \sin \frac{\theta}{2} & \cos \frac{\theta}{2}+i \sin \psi \sin \frac{\theta}{2}
\end{array}\right) .
$$

A rotation of $\mathbb{S}^{3}$ about the generic fibre $F(t)$ through angle $\omega$ has the form $\langle R(\omega), R(\omega)\rangle$, where

$$
R(\omega)=\left(\begin{array}{cc}
\cos \frac{\omega}{2} & i \sin \frac{\omega}{2} \\
i \sin \frac{\omega}{2} & \cos \frac{\omega}{2}
\end{array}\right)
$$

The image of $F(t)$ under the Hopf map $h$ is $(0,0)$ w.r.t. the polar coordinates. The following lemma shows how to obtain a rotation about the pre-image $h^{-1}(\psi, \theta)$ of an arbitrary point $(\psi, \theta)$.

LEMMA 2. A rotation through angle $\omega$ about an axis $C(t)$ in $\mathbb{S}^{3}$ which is the pre-image of a point $(\psi, \theta) \in \mathbb{S}^{2}$ with respect to the Hopf map is

$$
\left\langle\overline{M(\psi, \theta)} R(\omega) M(\psi, \theta)^{t}, R(\omega)\right\rangle .
$$

Proof. Since we have that $C(t)=M(\psi, \theta) F(t)$ and $R(\omega)^{t} F(t) \overline{R(\omega)}=F(t)$ for every $0 \leq t \leq 2 \pi$, then

$$
\begin{aligned}
\left(\overline{M(\psi, \theta)} R(\omega) M(\psi, \theta)^{t}\right)^{t} C(t) \overline{R(\omega)} & =M(\psi, \theta) R(\omega)^{t} F(t) \overline{R(\omega)} \\
=M(\psi, \theta) F(t) & =C(t)
\end{aligned}
$$

by a straightforward computation. Here, we use the fact that $M(\psi, \theta) \in S U_{2}(\mathbb{C})$, and so $\overline{M(\psi, \theta)^{t}} M(\psi, \theta)=\mathrm{id}$.

Another remarkable property of the Hopf fibration is discussed below. 
Lemma 3. Every two fibres $C_{1}(t)$ and $C_{2}(t)$ of the Hopf fibration are equidistant geodesic lines (great circles) in $\mathbb{S}^{3}$.

If $C_{i}(t), i \in\{1,2\}$ are pre-images of the points $\widehat{C}_{i} \in \mathbb{S}^{2}$, then the length $\delta$ of the common perpendicular for $C_{1}(t)$ and $C_{2}(t)$ equals $\frac{1}{2} d\left(\widehat{C}_{1}, \widehat{C}_{2}\right)$.

Proof. The proof follows from the fact that the Hopf fibration is a Riemannian submersion between $\mathbb{S}^{3}$ and $\mathbb{S}_{\frac{1}{2}}^{2}=\left\{(x, y, z) \in \mathbb{R}^{3} \mid x^{2}+y^{2}+z^{2}=\frac{1}{4}\right\}$ with their standard Riemannian metrics of sectional curvature +1 and +4 , respectively (see Proposition 1.1 and Proposition 1.2 of [9]).

Every rotation about a fibre of the Hopf fibration induces a rotation about a point of its base.

LEMMA 4. Given a rotation $\langle A, B\rangle \in S U_{2}(\mathbb{C}) \times S U_{2}(\mathbb{C})$ about a fibre $C(t)$ of the Hopf fibration, the transformation $A \in S U_{2}(\mathbb{C})$ induces a rotation of $\mathbb{S}^{2}$ about the point to which $C(t)$ projects under the Hopf map.

Proof. Rotation about the fibre $C(t)=M(\psi, \theta) F(t)$ which projects to the point $(\psi, \theta) \in \mathbb{S}^{2}$ has the form

$$
\langle A, B\rangle=\left\langle\overline{M(\psi, \theta)} R(\omega) M(\psi, \theta)^{t}, R(\omega)\right\rangle .
$$

Observe that the rotation $\langle R(\omega), R(\omega)\rangle$ fixes the geodesic $F(t)$ in $\mathbb{S}^{3}$ and $R(\omega)$ fixes the point $\widehat{F}=\left(\begin{array}{cc}0 & i \\ i & 0\end{array}\right)$ in $\mathbb{S}^{2}$. Thus, $A \in S U_{2}(\mathbb{C})$ fixes the point $\widehat{C}=M(\psi, \theta) \widehat{F} \overline{M(\psi, \theta)^{t}}$. By a straightforward computation, we obtain that

$$
\widehat{C}=\left(\begin{array}{cc}
i \cos \psi \sin \theta & \sin \theta \sin \psi+i \cos \theta \\
-\sin \theta \sin \psi+i \cos \theta & -i \cos \psi \sin \theta
\end{array}\right)
$$

The point $\widehat{C} \in \mathbb{S}^{2}$ corresponds to $(\psi, \theta)$ w.r.t. the polar coordinates.

4. Examples of rigidity and flexibility. In this section, we work out two principal examples of Seifert fibred cone-manifolds: the first represents a rigid cone-manifold, the second one is flexible.

4.1. Case of rigidity: the cone-manifold $\mathcal{H}_{3}(\alpha, \beta, \gamma)$. Let $\mathcal{H}_{3}(\alpha, \beta, \gamma)$ denote a three-dimensional cone-manifold with underlying space the sphere $\mathbb{S}^{3}$ and singular locus formed by the link $\mathcal{H}_{3}$ with cone angles $\alpha, \beta$ and $\gamma$ along its components. The remaining discussion is devoted to the proof of

THEOREM 2. The cone-manifold $\mathcal{H}_{3}(\alpha, \beta, \gamma)$ admits a spherical structure if the following inequalities are satisfied:

$$
\begin{gathered}
2 \pi-\gamma<\alpha+\beta<2 \pi+\gamma, \\
-2 \pi+\gamma<\alpha-\beta<2 \pi-\gamma .
\end{gathered}
$$

The spherical structure on $\mathcal{H}_{3}(\alpha, \beta, \gamma)$ is unique (i.e. $\mathcal{H}_{3}(\alpha, \beta, \gamma)$ is globally rigid).

The lengths $\ell_{\alpha}, \ell_{\beta}, \ell_{\gamma}$ of its singular strata are pairwise equal and the following formula holds:

$$
\ell_{\alpha}=\ell_{\beta}=\ell_{\gamma}=\frac{\alpha+\beta+\gamma}{2}-\pi .
$$




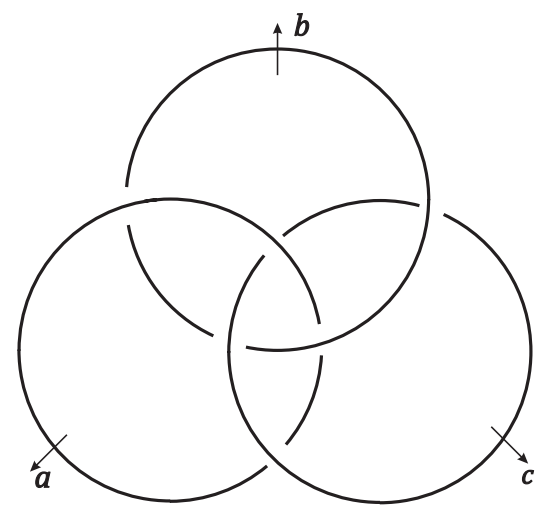

Figure 2. The link $\mathcal{H}_{3}$.

The volume of $\mathcal{H}_{3}(\alpha, \beta, \gamma)$ equals

$$
\operatorname{Vol} \mathcal{H}_{3}(\alpha, \beta, \gamma)=\frac{1}{2}\left(\frac{\alpha+\beta+\gamma}{2}-\pi\right)^{2} .
$$

Proof. First, we construct a holonomy map for $\mathcal{H}_{3}(\alpha, \beta, \gamma)$. By applying Wirtinger's algorithm, one obtains the following fundamental group presentation for the link $\mathcal{H}_{3}$ (see Figure 2):

$$
\Gamma=\pi_{1}\left(\mathbb{S}^{3} \backslash \mathcal{H}_{3}\right)=\langle a, b, c, h \mid a c b=b a c=c b a=h, h \in Z(\Gamma)\rangle,
$$

that is a central extension by $h$ of the thrice-punctured sphere group

$$
\Gamma_{0}=\pi_{1}\left(\mathbb{S}^{2} \backslash\{3 \text { points }\}\right)=\langle a, b, c \mid a c b=b a c=c b a=\mathrm{id}\rangle .
$$

Consider a holonomy map

$$
\rho: \Gamma \longmapsto \text { Isom }^{+} \mathbb{S}^{3} \cong S O_{4}(\mathbb{R}) .
$$

Let $\widetilde{\rho}$ denote its lift to $S U_{2}(\mathbb{C}) \times S U_{2}(\mathbb{C})$, which is a two-fold covering of $S O_{4}(\mathbb{R})$ (see [7]):

$$
\widetilde{\rho}=\left\langle\widetilde{\rho}_{1}, \widetilde{\rho}_{2}\right\rangle: \Gamma \longmapsto S U_{2}(\mathbb{C}) \times S U_{2}(\mathbb{C}) .
$$

Let us note, that if holonomy images of any two generators of $\Gamma$ commute, then the whole homomorphic image $\widetilde{\rho}(\Gamma)$ is abelian. Thus, for a representation $\tilde{\rho}$ we have the following three cases, up to a suitable conjugation, are possible:

(i) $\tilde{\rho}=\left(\widetilde{\rho}_{1}, \widetilde{\rho}_{2}\right): \Gamma \rightarrow S U_{2}(\mathbb{C}) \times S U_{2}(\mathbb{C})$, both $\widetilde{\rho}_{1}$ and $\tilde{\rho}_{2}$ are non-abelian,

(ii) $\tilde{\rho}: \Gamma \rightarrow \mathbb{S}^{1} \times \mathbb{S}^{1}$, an abelian representation,

(iii) $\widetilde{\rho}=\left(\widetilde{\rho}_{1}, \widetilde{\rho}_{2}\right): \Gamma \rightarrow S U_{2}(\mathbb{C}) \times \mathbb{S}^{1}$, where $\widetilde{\rho}_{1}$ is non-abelian.

For case (i), let us first suppose that $\tilde{\rho}(h)$ is non-trivial. Since the holonomy images of the meridians $a, b$ and $c$ have to commute with the holonomy image of $h$, they are simultaneously diagonalisable. We arrive at case (ii).

If $\tilde{\rho}(h)$ is trivial, then we have two non-abelian representations $\tilde{\rho}_{i}: \Gamma_{0} \rightarrow S U_{2}(\mathbb{C})$. Since the holonomy images of the meridians correspond to rotations along geodesic 
lines in $\mathbb{S}^{3}$, it follows by [2, Lemma 9.2] that $\operatorname{tr} \widetilde{\rho}_{1}(x)=\operatorname{tr} \widetilde{\rho}_{2}(x)$ for $x \in\{a, b, c\}$. The base space of the fibred cone-manifold $\mathcal{H}_{3}(\alpha, \beta, \gamma)$ is a turnover $\mathbb{S}^{2}(\alpha, \beta, \gamma)$, with $\alpha$, $\beta, \gamma$ cone angles. Then, by [10, Lemma 4.1], up to a conjugation, $\widetilde{\rho}=\left(\widetilde{\rho}_{1}, \widetilde{\rho}_{1}\right)$. The representation $\rho: \Gamma \rightarrow S O(4)$ is conjugate into $S O(3)$ and the holonomy images of the meridians have a common fixed point in $\mathbb{S}^{3}$. Thus, their axis intersect, which does not correspond to a non-degenerate spherical structure on the cone-manifold $\mathcal{H}_{3}(\alpha, \beta, \gamma)$.

For case (ii), up to a suitable conjugation, the representation $\tilde{\rho}$ preserves the Hopf fibration. Thus, by Lemma 4 , it descends to an abelian representation of $\Gamma_{0}$, which cannot be a holonomy of a non-degenerate spherical structure on the base of the fibration.

Finally, case (iii) is left. By [2, Lemma 9.2], one has

$$
\begin{aligned}
& \tilde{\rho}(a)=\left\langle m_{a}^{t} R(\alpha) \overline{m_{a}}, R(\alpha)\right\rangle, \\
& \widetilde{\rho}(b)=\left\langle m_{b}^{t} R(\beta) \overline{m_{b}}, R(\beta)\right\rangle, \\
& \widetilde{\rho}(c)=\left\langle m_{c}^{t} R(\gamma) \overline{m_{c}}, R(\gamma)\right\rangle
\end{aligned}
$$

for $m_{a}, m_{b}, m_{c} \in S U_{2}(\mathbb{C})$.

Note, that every matrix $m \in S U_{2}(\mathbb{C})$ is of the form $m=R(\tau) M(\psi, \theta)$ for suitable $0 \leq \psi \leq \pi, 0 \leq \theta, \tau \leq 2 \pi$. Then, we obtain that the image of every meridian in $\Gamma=$ $\pi_{1}\left(\mathbb{S}^{3} \backslash \mathcal{H}_{3}\right)$ has the form

$$
\begin{gathered}
\left\langle m^{t} R(\omega) \bar{m}, R(\omega)\right\rangle=\left\langle M^{t}(\psi, \theta) R^{t}(\tau) R(\omega) \overline{R(\tau)} \overline{M(\psi, \tau)}, R(\omega)\right\rangle \\
=\left\langle M^{t}(\psi, \theta) R(\omega) \overline{M(\psi, \theta)}, R(\omega)\right\rangle,
\end{gathered}
$$

since $R(\omega)$ and $R(\tau)$ commute. Hence, Lemma 2 implies that every meridian is mapped by $\widetilde{\rho}$ to a rotation about an appropriate fibre of the Hopf fibration. By Propositions 2.1 and 2.2 of [9], the holonomy preserves the fibration structure.

Let $A=\widetilde{\rho}(a), B=\widetilde{\rho}(b), C=\widetilde{\rho}(c)$ be holonomy images of the generators $a, b, c$ for $\Gamma=\pi_{1}\left(\mathbb{S}^{3} \backslash \mathcal{H}_{3}\right)$.

After a suitable conjugation in $S U_{2}(\mathbb{C}) \times S U_{2}(\mathbb{C})$, we obtain

$$
\begin{aligned}
& A=\left\langle A_{l}, A_{r}\right\rangle=\langle R(\alpha), R(\alpha)\rangle, \\
& B=\left\langle B_{l}, B_{r}\right\rangle=\left\langle\overline{M(0, \phi)} R(\beta) M(0, \phi)^{t}, R(\beta)\right\rangle, \\
& C=\left\langle C_{l}, C_{r}\right\rangle=\left\langle\overline{M(\psi, \theta)} R(\gamma) M(\psi, \theta)^{t}, R(\gamma)\right\rangle .
\end{aligned}
$$

In order for the holonomy map $\widetilde{\rho}$ to be a homomorphism, the following relations should hold:

$$
\begin{aligned}
& A_{l} C_{l} B_{l}=B_{l} A_{l} C_{l}=C_{l} B_{l} A_{l}, \\
& A_{r} C_{r} B_{r}=B_{r} A_{r} C_{r}=C_{r} B_{r} A_{r} .
\end{aligned}
$$

The latter of them is satisfied by the construction of $\widetilde{\rho}: \Gamma \rightarrow S U_{2}(\mathbb{C}) \times \mathbb{S}^{1}$.

Let us consider the former relations. By Lemma 4, the elements $A_{l}, B_{l}$ and $C_{l}$ are rotations of $\mathbb{S}^{2}$ about the points $\widehat{F}_{a}=(0,0), \widehat{F}_{b}=(0, \phi)$ and $\widehat{F}_{c}=(\psi, \theta)$, respectively. Since $\widehat{F}_{a}, \widehat{F}_{b}, \widehat{F}_{c}$ form a triangle on $\mathbb{S}^{2}$ and the base space of $\mathcal{H}_{3}(\alpha, \beta, \gamma)$ is a turnover with $\alpha, \beta, \gamma$ cone angles, one may expect the following

LEMMA 5. The points $\widehat{F}_{a}=(0,0), \widehat{F}_{b}=(0, \phi)$ and $\widehat{F}_{c}=(\psi, \theta)$ form a triangle with angles $\frac{\alpha}{2}, \frac{\beta}{2}$ and $\frac{\gamma}{2}$ at the corresponding vertices. 
Proof. By a straightforward computation, we obtain that

$$
\begin{aligned}
& A_{l} C_{l} B_{l}-B_{l} A_{l} C_{l}=\left(\begin{array}{cc}
i R_{1} & R_{2}+i R_{3} \\
-R_{2}+i R_{3} & -i R_{1}
\end{array}\right), \\
& C_{l} B_{l} A_{l}-B_{l} A_{l} C_{l}=\left(\begin{array}{cc}
i R_{4} & R_{5}+i R_{3} \\
-R_{5}+i R_{3} & -i R_{4}
\end{array}\right),
\end{aligned}
$$

where

$$
\begin{aligned}
R_{1}= & 2 \sin \frac{\beta}{2} \sin \frac{\gamma}{2} \sin \theta \cos \phi \sin \left(\frac{\alpha}{2}-\psi\right) \\
R_{2}= & 2 \sin \frac{\beta}{2}\left(\cos \frac{\gamma}{2} \sin \frac{\alpha}{2} \sin \phi+\sin \frac{\gamma}{2}\left(-\cos \phi \cos \left(\frac{\alpha}{2}-\psi\right) \sin \theta\right.\right. \\
& \left.\left.+\cos \frac{\alpha}{2} \cos \theta \sin \phi\right)\right) \\
R_{3}= & -2 \sin \frac{\beta}{2} \sin \frac{\gamma}{2} \sin \theta \sin \phi \sin \left(\frac{\alpha}{2}-\psi\right), \\
R_{4}= & 2 \sin \frac{\gamma}{2}\left(\cos \theta \sin \frac{\alpha}{2} \sin \frac{\beta}{2} \sin \phi-\left(\cos \frac{\beta}{2} \sin \frac{\alpha}{2}\right.\right. \\
& \left.\left.+\cos \frac{\alpha}{2} \sin \frac{\beta}{2} \cos \phi\right) \sin \theta \sin \psi\right), \\
R_{5}= & 2 \sin \frac{\gamma}{2}\left(\cos \frac{\beta}{2} \cos \psi \sin \frac{\alpha}{2} \sin \theta\right. \\
& \left.+\cos \frac{\alpha}{2} \sin \frac{\beta}{2}(\cos \phi \cos \psi \sin \theta-\cos \theta \sin \phi)\right)
\end{aligned}
$$

In order to determine the parameters $\phi, \psi$ and $\theta$, one can proceed as follows: these are determined by the system of equations $R_{k}=0, k \in\{1, \ldots, 5\}$ under the restrictions $0<\alpha, \beta, \gamma<2 \pi$ and $0<\psi \leq 2 \pi, 0<\theta \leq \pi$. Thus, the common solutions to $R_{1}$ and $R_{3}$ are $\psi=\frac{\alpha}{2}$ and $\psi=\frac{\alpha}{2} \pm \pi$. We claim that the cone angles in the base space of $\mathcal{H}_{3}(\alpha, \beta, \gamma)$ and along its fibres are the same, and choose $\psi=\frac{\alpha}{2}$.

Taking into account that $0<\alpha, \beta, \gamma<2 \pi$ (this implies that the sine functions of half cone angles are non-zero), turn the set of relations $R_{k}, k \in\{1, \ldots, 5\}$ into a new one:

$$
\begin{aligned}
& \widetilde{R}_{1}=-\cos \phi \sin \frac{\gamma}{2} \sin \theta+\left(\sin \frac{\alpha}{2} \cos \frac{\gamma}{2}+\cos \frac{\alpha}{2} \sin \frac{\gamma}{2} \cos \theta\right) \sin \phi \\
& \widetilde{R}_{2}=-\cos \theta \sin \frac{\beta}{2} \sin \phi+\left(\sin \frac{\alpha}{2} \cos \frac{\beta}{2}+\cos \frac{\alpha}{2} \sin \frac{\beta}{2} \cos \phi\right) \sin \theta
\end{aligned}
$$

Note, that the conditions of Theorem 2 concerning cone angles are exactly the existence conditions for a spherical triangle with angles $\frac{\alpha}{2}, \frac{\beta}{2}$ and $\frac{\gamma}{2}$. For the latter, the following trigonometric identities (spherical cosine and sine rules) are satisfied [25, 
Theorems 2.5.2 and 2.5.4]:

$$
\begin{aligned}
\cos \phi= & \frac{\cos \frac{\gamma}{2}+\cos \frac{\alpha}{2} \cos \frac{\beta}{2}}{\sin \frac{\alpha}{2} \sin \frac{\beta}{2}}, \\
\cos \theta= & \frac{\cos \frac{\beta}{2}+\cos \frac{\alpha}{2} \cos \frac{\gamma}{2}}{\sin \frac{\alpha}{2} \sin \frac{\gamma}{2}}, \\
& \frac{\sin \phi}{\sin \frac{\gamma}{2}}=\frac{\sin \theta}{\sin \frac{\beta}{2}} .
\end{aligned}
$$

These identities state that the points $\widehat{F}_{a}, \widehat{F}_{b}$ and $\widehat{F}_{c}$ form a triangle on $\mathbb{S}^{2}$ with angles $\frac{\alpha}{2}, \frac{\beta}{2}$ and $\frac{\gamma}{2}$ at the corresponding vertices. Its double provides the base turnover with cone angles $\alpha, \beta$ and $\gamma$ for the fibred cone-manifold $\mathcal{H}_{3}(\alpha, \beta, \gamma)$.

On substituting the expressions for $\cos \phi$ and $\cos \psi$ above in the relations $\widetilde{R}_{k}$, $k \in\{1,2\}$ and taking into account the sine rule, one obtains that $\widetilde{R}_{k}=0, k \in\{1,2\}$. The lemma is proven.

Let $\mathcal{S}$ denote the domain of cone angles indicated in the statement of the theorem:

$$
\mathcal{S}=\left\{\begin{array}{l|l}
\vec{\alpha}=(\alpha, \beta, \gamma) & \begin{array}{c}
2 \pi-\gamma<\alpha+\beta<2 \pi+\gamma \\
-2 \pi+\gamma<\alpha-\beta<2 \pi-\gamma
\end{array}
\end{array}\right\} .
$$

Let $\mathcal{S}^{*}$ denote the subset of $\mathcal{S}$, such that for every triple of cone angles $\vec{\alpha}=(\alpha, \beta, \gamma) \in \mathcal{S}^{*}$ there exists a spherical structure on $\mathcal{H}_{3}(\vec{\alpha})$. Our next step is to show that $\mathcal{S}^{*}$ coincides with $\mathcal{S}$.

The set $\mathcal{S}^{*}$ is non-empty. From [8], it follows that $\mathcal{H}_{3}(\pi, \pi, \pi)$ has a spherical structure. The orbifold $\mathcal{H}_{3}(\pi, \pi, \pi)$ is Seifert fibred and its base is a turnover with cone angles equal to $\pi$. Thus, the point $(\pi, \pi, \pi) \in \mathcal{S}$ belongs to $\mathcal{S}^{*}$.

The set $\mathcal{S}^{*}$ is open, because a deformation of the holonomy induces a deformation of the structure [20].

In order to prove that the set $\mathcal{S}^{*}$ is closed, we consider a sequence $\vec{\alpha}_{n}=\left(\alpha_{n}, \beta_{n}, \gamma_{n}\right)$ in $\mathcal{S}^{*}$ converging to $\vec{\alpha}_{\infty}=\left(\alpha_{\infty}, \beta_{\infty}, \gamma_{\infty}\right)$ in $\mathcal{S}$. Since every spherical cone-manifold with cone angles $\leq 2 \pi$ is an Alexandrov space with curvature $\geq 1$ [3], we obtain that the diameter of $\overline{\mathcal{H}}_{3}\left(\vec{\alpha}_{n}\right)$ is bounded above: $\operatorname{diam} \mathcal{H}_{3}\left(\vec{\alpha}_{n}\right) \leq \pi$.

Let dist $\mathcal{H}_{3}\left(\vec{\alpha}_{n}\right)$ denote the minimum of the mutual distances between the axis of rotations $A, B$ and $C$. Since $\vec{\alpha}_{\infty} \in \mathcal{S}$, we have by Lemma 5 that the turnover $\mathbb{S}^{2}\left(\vec{\alpha}_{\infty}\right)$ is non-degenerate. By making use of Lemma 3, one obtains that (restricting to a subsequence, if needed) for every $\vec{\alpha}_{n} \in \mathcal{S}, n=1,2, \ldots$ the function dist $\mathcal{H}_{3}\left(\vec{\alpha}_{n}\right)$ is uniformly bounded below away from zero:

$$
\text { dist } \mathcal{H}_{3}\left(\vec{\alpha}_{n}\right) \geq d_{0}>0, \quad n=1,2, \ldots
$$

Then, we use the following facts [3]:

(1) The Gromov-Hausdorff limit of Alexandrov spaces with curvature $\geq 1$, dimension $=3$ and bounded diameter is an Alexandrov space with curvature $\geq 1$ and dimension $\leq 3$,

(2) Dimension of an Alexandrov space with curvature $\geq 1$ holds the same at every point (the word 'dimension' means Hausdorff or topological dimension, which are equal in the case of curvature $\geq 1$ ). 
Since dist $\mathcal{H}_{3}\left(\vec{\alpha}_{n}\right) \geq d_{0}>0$, the sequence $\mathcal{H}_{3}\left(\vec{\alpha}_{n}\right)$ does not collapse. Thus, the cone-manifold $\mathcal{H}_{3}\left(\vec{\alpha}_{\infty}\right)$ has a non-degenerate spherical structure and $\vec{\alpha}_{\infty} \in \mathcal{S}^{*}$.

The subset $\mathcal{S}^{*} \subset \mathcal{S}$ is non-empty, as well as both closed and open. This implies $\mathcal{S}^{*}=\mathcal{S}$.

Finally, we claim the following fact concerning the geometric characteristics of $\mathcal{H}_{3}(\alpha, \beta, \gamma)$ cone-manifold:

LEMMA 6. Let $\ell_{\alpha}, \ell_{\beta}, \ell_{\gamma}$ denote the lengths of the singular strata for $\mathcal{H}_{3}(\alpha, \beta, \gamma)$ cone-manifold with cone angles $\alpha, \beta$ and $\gamma$. Then,

$$
\ell_{\alpha}=\ell_{\beta}=\ell_{\gamma}=\frac{\alpha+\beta+\gamma}{2}-\pi
$$

The volume of $\mathcal{H}_{3}(\alpha, \beta, \gamma)$ is

$$
\operatorname{Vol} \mathcal{H}_{3}(\alpha, \beta, \gamma)=\frac{1}{2}\left(\frac{\alpha+\beta+\gamma}{2}-\pi\right)^{2} .
$$

Proof. Let us calculate the geometric parameters explicitly, using the holonomy map defined above. First, we introduce two notions suitable for the further discussion. Given an element $M=\left\langle M_{l}, M_{r}\right\rangle \in S U_{2}(\mathbb{C}) \times S U_{2}(\mathbb{C})$, one may assume that the pair of matrices $\left\langle M_{l}, M_{r}\right\rangle$ is conjugated, by means of a certain element $\left\langle C_{l}, C_{r}\right\rangle \in S U_{2}(\mathbb{C}) \times$ $S U_{2}(\mathbb{C})$, to the pair of diagonal matrices

$$
\left\langle\left(\begin{array}{cc}
e^{i \gamma} & 0 \\
0 & e^{-i \gamma}
\end{array}\right),\left(\begin{array}{cc}
e^{i \varphi} & 0 \\
0 & e^{-i \varphi}
\end{array}\right)\right\rangle
$$

with $0 \leq \gamma, \varphi \leq \pi$.

Then, call the translation length of $M$ the quantity $\delta(M):=\varphi-\gamma$ and call the 'jump' of $M$ the quantity $\nu(M):=\varphi+\gamma$ (see [11] and [31, chapter 6.4.2]). We suppose that $\varphi>\gamma$, otherwise changing $\gamma, \varphi$ for $2 \pi-\gamma$ and $\pi-\varphi$ makes the considered tuple to have the desired form.

Recall that the representation of $\Gamma=\pi_{1}\left(\mathbb{S}^{3} \backslash \mathcal{H}_{3}\right)$ is

$$
\Gamma=\langle a, b, c, h \mid a c b=b a c=c b a=h, h \in Z(\Gamma)\rangle,
$$

where $a, b, c$ are meridians and $h$ is a longitudinal loop that represents a fibre. Denote by $H$ the image of $h$ under the holonomy map $\widetilde{\rho}$. Then, we obtain

$$
\ell_{\alpha}=\ell_{\beta}=\ell_{\gamma}=\delta(H)
$$

Since $A=\widetilde{\rho}(a)$ and $H=\widetilde{\rho}(h)$ commute, there exists an element $C=\left\langle C_{l}, C_{r}\right\rangle$ of $S U_{2}(\mathbb{C}) \times S U_{2}(\mathbb{C})$ such that

$$
\begin{gathered}
C A C^{-1}=\left\langle\left(\begin{array}{cc}
e^{i \frac{\alpha}{2}} & 0 \\
0 & e^{-i \frac{\alpha}{2}}
\end{array}\right),\left(\begin{array}{cc}
e^{i \frac{\alpha}{2}} & 0 \\
0 & e^{-i \frac{\alpha}{2}}
\end{array}\right)\right\rangle, \\
C H C^{-1}=\left\langle\left(\begin{array}{cc}
e^{i \gamma(H)} & 0 \\
0 & e^{-i \gamma(H)}
\end{array}\right),\left(\begin{array}{cc}
e^{i \varphi(H)} & 0 \\
0 & e^{-i \varphi(H)}
\end{array}\right)\right\rangle .
\end{gathered}
$$


By a straightforward computation similar to that in Lemma 5, one obtains

$$
2 \cos \gamma(H)=\operatorname{tr} H_{l}=\operatorname{tr} A_{l} C_{l} B_{l}=\operatorname{tr}(-\mathrm{id})=2 \cos \pi
$$

and

$$
2 \cos \varphi(H)=\operatorname{tr} H_{r}=\operatorname{tr} A_{r} C_{r} B_{r}=2 \cos \frac{\alpha+\beta+\gamma}{2} .
$$

From the foregoing discussion, the singular stratum's length is

$$
\ell_{\alpha}=\delta(H)=\frac{\alpha+\beta+\gamma}{2}-\pi
$$

An analogous equality holds for $\ell_{\beta}$ and $\ell_{\gamma}$.

By the Schläfli formula [12], the following relation holds:

$$
2 \mathrm{dVol} \mathcal{H}_{3}(\alpha, \beta, \gamma)=\ell_{\alpha} \mathrm{d} \alpha+\ell_{\beta} \mathrm{d} \beta+\ell_{\gamma} \mathrm{d} \gamma
$$

Solving this differential equality, we obtain that

$$
\operatorname{Vol} \mathcal{H}_{3}(\alpha, \beta, \gamma)=\frac{1}{2}\left(\frac{\alpha+\beta+\gamma}{2}-\pi\right)^{2}+\operatorname{Vol}_{0},
$$

where $\mathrm{Vol}_{0}$ is an arbitrary constant. Since the geometric structure on the base space of the fibration (consequently, on the whole $\mathcal{H}_{3}(\alpha, \beta, \gamma)$ cone-manifold) degenerates when $\alpha+\beta+\gamma \longrightarrow 2 \pi$, the equality $\operatorname{Vol}_{0}=0$ follows from the volume function continuity.

Consider a holonomy $\tilde{\rho}=\left\langle\widetilde{\rho}_{1}, \tilde{\rho}_{2}\right\rangle: \Gamma=\pi_{1}\left(\mathbb{S}^{3} \backslash \mathcal{H}_{3}\right) \rightarrow S U_{2}(\mathbb{C}) \times S U_{2}(\mathbb{C})$ for $\mathcal{H}_{3}(\alpha, \beta, \gamma)$ cone-manifold. As we already know from the preceding discussion, one has $\tilde{\rho}: \Gamma \rightarrow S U_{2}(\mathbb{C}) \times \mathbb{S}^{1}$ essentially, and $\widetilde{\rho}_{1}$ determines $\widetilde{\rho}_{2}$ up to a conjugation by means of the equality $\operatorname{tr} \widetilde{\rho}_{1}(m)=\operatorname{tr} \widetilde{\rho}_{2}(m)$ for meridians in $\Gamma$. So any deformation of $\widetilde{\rho}$ is a deformation of $\tilde{\rho}_{1}$. In the case of $\mathcal{H}_{3}(\alpha, \beta, \gamma)$, the map $\tilde{\rho}_{1}$ is a non-abelian representation of the base turnover group. Spherical turnover is rigid, that means $\widetilde{\rho}_{1}$ is determined only by the corresponding cone angles. Thus, $\mathcal{H}_{3}(\alpha, \beta, \gamma)$ is locally rigid.

The global rigidity follows from the fact that every $\mathcal{H}_{3}(\alpha, \beta, \gamma)$ cone-manifold could be deformed to the orbifold $\mathcal{H}_{3}(\pi, \pi, \pi)$ by a continuous path through locally rigid structures. This assertion holds since $\mathcal{S}^{*}$ contains the point $(\pi, \pi, \pi)$ and $\mathcal{S}^{*}$ is convex. The global rigidity of $\mathcal{H}_{3}(\pi, \pi, \pi)$ spherical orbifold follows from $[\mathbf{2 6}, 27]$ and implies the global rigidity of $\mathcal{H}_{3}(\alpha, \beta, \gamma)$ by means of deforming the orbifold structure backwards to the considered cone-manifold one.

4.2. Case of flexibility: the cone-manifold $\mathcal{H}_{4}(\alpha)$. Let $\mathcal{H}_{4}(\alpha)$ denote a threedimensional cone-manifold with underlying space the sphere $\mathbb{S}^{3}$ and singular locus formed by the link $\mathcal{H}_{4}$ with cone angle $\alpha$ along all its components (see Figure 3 ).

The following theorem provides an example of a flexible cone-manifold, which is Seifert fibred.

THEOREM 3. The cone-manifold $\mathcal{H}_{4}(\alpha)$ admits a spherical structure if

$$
\pi<\alpha<2 \pi \text {. }
$$




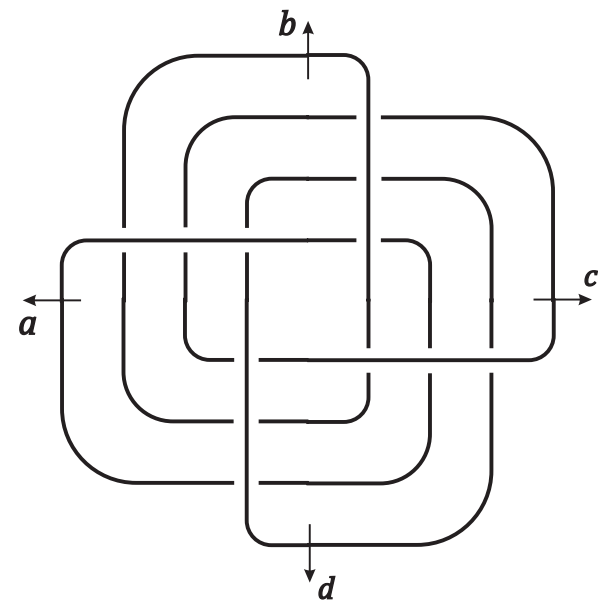

Figure 3. The link $\mathcal{H}_{4}$.

This structure is not unique (i.e. $\mathcal{H}_{4}(\alpha)$ is not globally, nor locally rigid). The deformation space contains an open interval that provides a one-parameter family of distinct spherical cone-metrics on $\mathbb{S}^{3}$.

The length of each singular stratum is

$$
\ell=2(\alpha-\pi)
$$

The volume of $\mathcal{H}_{4}(\alpha)$ equals

$$
\operatorname{Vol} \mathcal{H}_{4}(\alpha)=2(\alpha-\pi)^{2} .
$$

Proof. The following lemma precedes the proof of the theorem.

LEMMA 7. Given a quadrangle $Q$ on $\mathbb{S}^{2}$ with three right angles and one angle $\frac{\alpha}{2}$ (see Figure 4), the following statements hold:

(1) The quadrangle $Q$ exists if $\pi<\alpha<2 \pi$,

(2) $\sin \ell_{1} \sin \ell_{2}=-\cos \frac{\alpha}{2}$,

(3) $\cos \phi=\frac{\cos \ell_{1} \cos \ell_{2}}{\sin \frac{\alpha}{2}}$,

(4) $\cos \psi=\tan \ell_{1} \cot \phi$,

(5) $0 \leq \ell_{1}, \ell_{2}, \phi, \psi \leq \frac{\pi}{2}$.

Proof. We refer the reader to $[30, \S 3.2]$ for a detailed proof of the statements above.

Given a quadrangle $Q$ from Lemma 7 (so-called Saccheri's quadrangle), one can construct another one, depicted in Figure 5, by reflecting $Q$ in its sides incident to the vertex $O$. We may regard $O$ to be the point $(0,0) \in \mathbb{S}^{2}$. Thus, the fibres over the corresponding vertices are

$$
\begin{aligned}
& F_{a}(t)=M(\psi, \phi) F(t), \\
& F_{b}(t)=M(\pi-\psi, \phi) F(t), \\
& F_{c}(t)=M(\pi+\psi, \phi) F(t), \\
& F_{d}(t)=M(2 \pi-\psi, \phi) F(t) .
\end{aligned}
$$




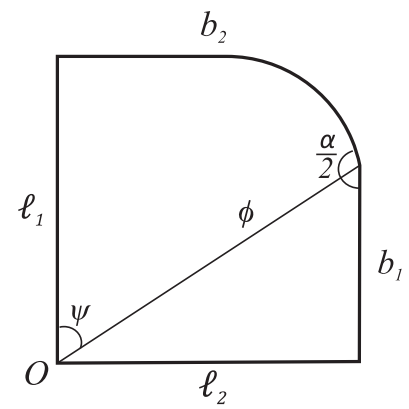

Figure 4. The quadrangle $Q$.

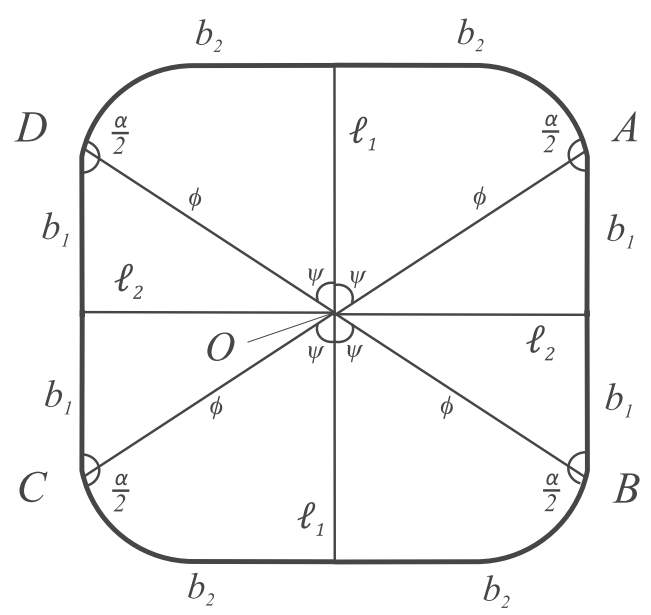

Figure 5. The base quadrangle $P$ for $\mathcal{H}_{4}(\alpha)$.

Let $A=\left\langle A_{l}, A_{r}\right\rangle, B=\left\langle B_{l}, B_{r}\right\rangle, C=\left\langle C_{l}, C_{r}\right\rangle, D=\left\langle D_{l}, D_{r}\right\rangle$ denote the respective rotations through angle $\alpha$ about the axis $F_{a}, F_{b}, F_{c}$ and $F_{d}$. From Lemma 2, one obtains

$$
\begin{aligned}
A_{l} & =\overline{M(\psi, \phi)} R(\alpha) M(\psi, \phi)^{t}, A_{r}=R(\alpha) ; \\
B_{l} & =\overline{M(\pi-\psi, \phi)} R(\alpha) M(\pi-\psi, \phi)^{t}, B_{r}=R(\alpha) ; \\
C_{l} & =\overline{M(\pi+\psi, \phi)} R(\alpha) M(\pi+\psi, \phi)^{t}, B_{r}=R(\alpha) ; \\
D_{l} & =\overline{M(2 \pi-\psi, \phi)} R(\alpha) M(2 \pi-\psi, \phi)^{t}, D_{r}=R(\alpha) .
\end{aligned}
$$

We assume that $\ell_{1}, \ell_{2}, \phi$ and $\psi$ satisfy the identities of Lemma 7.

The fundamental group of $\pi_{1}\left(\mathbb{S}^{3} \backslash \mathcal{H}_{4}\right)$ has the presentation

$$
\Gamma=\pi_{1}\left(\mathbb{S}^{3} \backslash \mathcal{H}_{4}\right)=\langle a, b, c, d, h \mid a d c b=b a d c=c b a d=d c b a=h, h \in Z(\Gamma)\rangle .
$$

Let us construct a lift of the holonomy map $\tilde{\rho}: \Gamma \rightarrow S U_{2}(\mathbb{C}) \times S U_{2}(\mathbb{C})$ as follows:

$$
\widetilde{\rho}(a)=A, \widetilde{\rho}(b)=B, \widetilde{\rho}(c)=C, \widetilde{\rho}(d)=D .
$$

Here, we choose $\tilde{\rho}: \Gamma \rightarrow S U_{2}(\mathbb{C}) \times \mathbb{S}^{1}$ by the same reason as in Theorem 2 . 


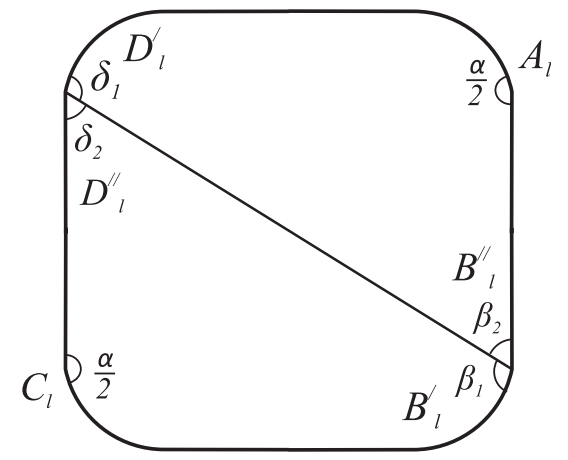

Figure 6. Section of $\mathrm{P}$ by the line joining vertices $B$ and $D$.

In order to show that the map $\tilde{\rho}$ is a homomorphism, one has to check whether the following relations are satisfied:

$$
\begin{aligned}
& A_{l} D_{l} C_{l} B_{l}=B_{l} A_{l} D_{l} C_{l}=C_{l} B_{l} A_{l} D_{l}=D_{l} C_{l} B_{l} A_{l}, \\
& A_{r} D_{r} C_{r} B_{r}=B_{r} A_{r} D_{r} C_{r}=C_{r} B_{r} A_{r} D_{r}=D_{r} C_{r} B_{r} A_{r} .
\end{aligned}
$$

The latter relations hold in view of the fact that the matrices $A_{r}, B_{r}, C_{r}$ and $D_{r}$ pairwise commute. Then, we show that the following equality holds:

$$
A_{l} D_{l} C_{l} B_{l}=\mathrm{id}
$$

To do this, split the quadrangle $P$ into two triangles by drawing a geodesic line from $B$ to $D$. Since $A_{l}, B_{l}, C_{l}$ and $D_{l}$ are rotations about the vertices of the quadrangle depicted in Figure 6., let us decompose the rotations $B_{l}=B_{l}^{\prime} B_{l}^{\prime \prime}$ and $D_{l}=D_{l}^{\prime} D_{l}^{\prime \prime}$ into the products of rotations $B_{l}^{\prime}, B_{l}^{\prime \prime}$ through angles $\beta_{1}, \beta_{2}$ and the rotations $D_{l}^{\prime}, D_{l}^{\prime \prime}$ through angles $\delta_{1}$ and $\delta_{2}$, respectively. The following equalities hold: $\beta_{1}+\beta_{2}=\frac{\alpha}{2}$ and $\delta_{1}+\delta_{2}=\frac{\alpha}{2}$. Thus, the triples $D_{l}^{\prime \prime}, C_{l}, B_{l}^{\prime}$ and $A_{l}, D_{l}^{\prime}, B_{l}^{\prime \prime}$ consist of rotations about the vertices of two disjoint triangles depicted in Figure 6. Similar to the computation of Lemma 6, we have

$$
D_{l}^{\prime \prime} C_{l} B_{l}^{\prime}=-\mathrm{id}
$$

and

$$
A_{l} D_{l}^{\prime} B_{l}^{\prime \prime}=-\mathrm{id}
$$

From the identities above, it follows that

$$
A_{l} D_{l} C_{l} B_{l}=A_{l} D_{l}^{\prime} D_{l}^{\prime \prime} C_{l} B_{l}^{\prime} B_{l}^{\prime \prime}=-A_{l} D_{l}^{\prime} B_{l}^{\prime \prime}=\mathrm{id} .
$$

The statement holds under a cyclic permutation of the factors. Thus,

$$
A_{l} D_{l} C_{l} B_{l}=B_{l} A_{l} D_{l} C_{l}=C_{l} B_{l} A_{l} D_{l}=D_{l} C_{l} B_{l} A_{l}=\mathrm{id} .
$$

Below we shall consider the side-length $\ell_{1}$ as a parameter. Let $\ell_{1}:=\tau$. Then, by Lemma 7, one has that $\sin \ell_{2}=-\frac{\cos \frac{\alpha}{2}}{\sin \tau}$ and $\ell_{2}:=\ell_{2}(\tau)$ is a well-defined continuous function of $\tau$. The quadrangle $P$ depends on the parameter $\tau$ continuously while keeping the angles in its vertices equal to $\frac{\alpha}{2}$. 
Let $\mathcal{H}_{4}(\alpha ; \tau)$ denote a three-dimensional cone-manifold with underlying space the sphere $\mathbb{S}^{3}$ and singular locus the link $\mathcal{H}_{4}$ with cone angle $\alpha$ along its components. Furthermore, its holonomy map is determined by the quadrangle $P$ described above (see Figure 5) depending on the parameter $\tau$. This means that the double of $P$ forms a 'pillowcase' cone-surface with all cone angles equal to $\alpha$, which is the base space for the fibred cone-manifold $\mathcal{H}_{4}(\alpha ; \tau)$.

Let $\mathbb{L}_{n}(\alpha, \beta)$ be a cone-manifold with underlying space the sphere $\mathbb{S}^{3}$ and singular locus a torus link of the type $(2,2 n)$ with cone angles $\alpha$ and $\beta$ along its components. Torus links of the type $(2,2 n)$ are two-bridge links. The corresponding cone-manifolds were previously considered in $[\mathbf{1 7}, \mathbf{2 2}]$. Since the cone-manifold $\mathcal{H}_{4}(\alpha)$ forms a four-fold branched covering of the cone-manifold $\mathbb{L}_{4}\left(\alpha, \frac{\pi}{2}\right)$, from [17, Theorem 2] we obtain that $\mathcal{H}_{4}(\alpha)$ has a spherical structure if $\pi<\alpha<2 \pi$. The length of each singular stratum equals to $\ell=2(\alpha-\pi)$ and the volume is $\operatorname{Vol}_{4}(\alpha)=2(\alpha-\pi)^{2}$.

Under the assumption that $\ell_{1}=\ell_{2}$, the base quadrangle depicted in Figure 5. appears to have a four-order symmetry. Moreover, by making use of Lemma 7, one may derive the following equalities: $\psi=\frac{\pi}{4}, \cos \phi=\cot \frac{\alpha}{4}$. The general formulas for the holonomy of $\mathcal{H}_{4}(\alpha)$ cone-manifold derived above subject to the condition $\ell_{1}=\ell_{2}$ (equivalently, the cone-manifold $\mathcal{H}_{4}(\alpha)$ has a four-order symmetry) give the holonomy map induced by the covering. Thus, $\mathcal{H}_{4}(\alpha) \cong \mathcal{H}_{4}\left(\alpha ; \arccos \left(\sqrt{2} \cos \frac{\alpha}{4}\right)\right)$ is a spherical cone-manifold.

We claim that one can vary the parameter $\tau$ in certain ranges while keeping spherical structure on $\mathcal{H}_{4}(\alpha ; \tau)$ non-degenerate.

LEMMA 8. If $\tau$ varies over $\left(\frac{\alpha-\pi}{2}, \frac{\pi}{2}\right)$, the cone-manifold $\mathcal{H}_{4}(\alpha ; \tau)$ has a non-degenerate spherical structure.

Proof. The proof has much in common with the proof of the spherical structure existence on $\mathcal{H}_{3}(\alpha, \beta, \gamma)$ cone-manifold given in Theorem 2. Let us express the identities of Lemma 7 in terms of the parameter $\ell_{1}:=\tau$. We obtain

$$
\begin{gathered}
\cos \phi=\cos \tau \sqrt{1-\cot ^{2} \frac{\alpha}{2} \cot ^{2} \tau}, \\
\cos \psi=\sqrt{\frac{1-\cot ^{2} \frac{\alpha}{2} \cot ^{2} \tau}{1+\cot ^{2} \frac{\alpha}{2} \cot ^{4} \tau}}, \\
\sin \ell_{2}=-\frac{\cos \frac{\alpha}{2}}{\sin \tau} .
\end{gathered}
$$

Since Lemma 7 states that $0 \leq \phi, \psi, \ell_{2} \leq \frac{\pi}{2}$, the functions $\phi:=\phi(\tau), \psi:=\psi(\tau)$, $\ell_{2}:=\ell_{2}(\tau)$ are well-defined and depend continuously on $\tau$.

Moreover, the following relations hold:

$$
\begin{aligned}
& \cos b_{1}=\frac{\cos \phi}{\cos \ell_{2}}=\cos \tau \sqrt{\frac{\sin ^{2} \tau-\cot ^{2} \frac{\alpha}{2} \cos ^{2} \tau}{\sin ^{2} \tau-\cos ^{2} \frac{\alpha}{2}}}, \\
& \cos b_{2}=\frac{\cos \phi}{\cos \tau}=\sqrt{1-\cot ^{2} \frac{\alpha}{2} \cot ^{2} \tau}
\end{aligned}
$$

If one sets the centre $O$ of the quadrangle $P$ to $(0,0) \in \mathbb{S}^{2}$, the whole quadrangle is situated in the upper hemisphere provided $\phi<\frac{\pi}{2}$. From the fact that $\cos b_{1} \geq \cos \phi$ and 
$\cos b_{2} \geq \cos \phi$, it follows $b_{1}, b_{2} \leq \phi$. Thus, $b_{1}, b_{2} \leq \frac{\pi}{2}$ and the functions $b_{1}:=b_{1}(\tau)$, $b_{2}:=b_{2}(\tau)$ are well-defined and continuous with respect to $\tau$.

Observe that if the condition $\frac{\alpha-\pi}{2}<\tau<\frac{\pi}{2}$ is satisfied, then the required inequality $\phi<\frac{\pi}{2}$ holds.

Let $\mathcal{S}_{\alpha}^{*}$ denote the subset of $\mathcal{S}_{\alpha}=\left\{\tau \mid \frac{\alpha-\pi}{2}<\tau<\frac{\pi}{2}\right\}$ that consists of the points $\tau \in \mathcal{S}_{\alpha}$ such that the cone-manifold $\mathcal{H}_{4}(\alpha ; \tau)$ has a non-degenerate spherical structure. We show $\mathcal{S}_{\alpha}^{*}=\mathcal{S}_{\alpha}$ by means of the fact that $\mathcal{S}_{\alpha}^{*}$ is both open and closed non-empty subset of $\mathcal{S}_{\alpha}$.

As noticed above, $\tau=\arccos \left(\sqrt{2} \cos \frac{\alpha}{4}\right)$ belongs to $\mathcal{S}_{\alpha}^{*}$. Hence, the set $\mathcal{S}_{\alpha}^{*}$ is nonempty.

The set $\mathcal{S}_{\alpha}^{*}$ is open by the fact that a deformation of the holonomy implies a deformation of the structure [20]. To prove that $\mathcal{S}_{\alpha}^{*}$ is closed, consider a sequence $\tau_{n}$ converging in $\mathcal{S}_{\alpha}^{*}$ to $\tau_{\infty} \in \mathcal{S}_{\alpha}$.

The lengths of common perpendiculars between the axis of rotations $A, B, C$ and $D$ defined above equal $b_{1}, b_{2}$ and $\phi$, respectively.

Since $\tau_{\infty}$ corresponds to a non-degenerated quadrangle, every cone-manifold $\mathcal{H}_{4}\left(\alpha ; \tau_{n}\right)$ has the quantities $b_{1}\left(\tau_{n}\right), b_{2}\left(\tau_{n}\right)$ and $\phi\left(\tau_{n}\right)$ uniformly bounded below away from zero. By the arguments similar to those of Theorem 2, we obtain that $\mathcal{H}_{4}\left(\alpha ; \tau_{\infty}\right)$ is a non-degenerate spherical cone-manifold. Thus, $\tau_{\infty}$ belongs to $\mathcal{S}_{\alpha}^{*}$. Hence, $\mathcal{S}_{\alpha}^{*}$ is closed.

Finally, we obtain that $\mathcal{S}_{\alpha}^{*}=\mathcal{S}_{\alpha}$. Thus, while $\tau$ varies over $\left(\frac{\alpha-\pi}{2}, \frac{\pi}{2}\right)$ the conemanifold $\mathcal{H}_{4}(\alpha ; \tau)$ does not collapse.

The following lemma shows that the interval $\left(\frac{\alpha-\pi}{2}, \frac{\pi}{2}\right)$ represents a part of the deformation space for possible spherical structures on $\mathcal{H}_{4}(\alpha ; \tau)$.

LEMMA 9. The cone-manifolds $\mathcal{H}_{4}\left(\alpha ; \tau_{1}\right)$ and $\mathcal{H}_{4}\left(\alpha ; \tau_{2}\right)$ with $\pi<\alpha<2 \pi$ and $\frac{\alpha-\pi}{2}<\tau_{1}, \tau_{2}<\frac{\pi}{2}$ are not isometric if $\tau_{1} \neq \tau_{2}$.

Proof. If the cone-manifolds $\mathcal{H}_{4}\left(\alpha ; \tau_{1}\right)$ and $\mathcal{H}_{4}\left(\alpha ; \tau_{2}\right)$ were isometric, then their holonomy maps $\tilde{\rho}_{i}, i=1,2$ would be conjugated representations of $\Gamma=\pi_{1}\left(\mathbb{S}^{3} \backslash \mathcal{H}_{4}\right)$ into $S U_{2}(\mathbb{C}) \times S U_{2}(\mathbb{C})$. Then, the mutual distances between the axis of rotations $A_{i}, B_{i}$, $C_{i}$ and $D_{i}, i=1,2$, coming from the holonomy maps $\widetilde{\rho}_{1}$ and $\widetilde{\rho}_{2}$ would be equal for the corresponding pairs. From Lemma 3 , it follows that the common perpendicular length for the given fibres $C_{1}$ and $C_{2}$ is half the distance between the images of $C_{1}$ and $C_{2}$ under the Hopf map. By applying Lemmas 3 and 8 to the base quadrangle $P$ of $\mathcal{H}_{4}\left(\alpha ; \tau_{i}\right), i=1,2$ one makes sure that the inequality $\tau_{1} \neq \tau_{2}$ implies the inequality for the lengths of corresponding common perpendiculars.

Note, that by the Schläfli formula the volume of $\mathcal{H}_{4}(\alpha)$ remains the same under any deformation preserving cone angles. Then, the formulas for the volume and the singular stratum length follow from the covering properties of $\mathcal{H}_{4}(\alpha) \stackrel{4: 1}{\rightarrow} \mathbb{L}_{4}\left(\alpha, \frac{\pi}{2}\right)$ and Theorem 2 of [17]. Thus, Theorem 3 is proven.

Acknowledgements. The author is grateful to Prof. J. Porti (Universitat Autònoma de Barcelona) and Prof. J.-M. Schlenker (Institut de Mathématiques de Toulouse) for their valuable comments on the paper and discussion of the subject. 


\section{REFERENCES}

1. M. Boileau, B. Leeb and J. Porti, Uniformization of small 3-orbifolds, C.R. Acad. Sci. Paris Se'r. I Math. 332(1) (2001), 57-62.

2. M. Boileau, B. Leeb and J. Porti, Geometrization of 3-dimensional orbifolds, Ann. Math. 162(1) (2005), 195-250.

3. Y. Burago, M. Gromov and G. Perelman, A. D. Aleksandrov spaces with curvature bounded below, Russian Math. Surveys 47 (1992), 1-58. 89-93.

4. G. Burde and K. Murasugi, Links and Seifert fiber spaces, Duke Math. J. 37(1) (1970),

5. A. Casson, An example of weak non-rigidity for cone manifolds with vertices, Talk at the Third MSJ regional workshop (Tokyo, 1998).

6. D. Cooper, C. Hodgson and S. Kerckhoff, Three-dimensional orbifolds and conemanifolds', vol. 5, Postface by S. Kojima. Tokyo: Mathematical Society of Japan, 2000. (MSJ Memoirs)

7. M. Culler, Lifting representations to covering groups, Adv. Math. 59(1) (1986), 64-70.

8. W. D. Dunbar, Geometric orbifolds, Rev. Mat. Univ. Complut. Madrid 1 (1988), 67-99.

9. H. Gluck and W. Ziller, The geometry of the Hopf fibrations, L'Enseign. Math. 32 (1986), 173-198.

10. W. Goldman, Ergodic theory on moduli spaces, Ann. Math. 146(3) (1997), 475-507.

11. H. M. Hilden, M. T. Lozano and J.-M. Montesinos-Amilibia, Volumes and ChernSimons invariants of cyclic coverings over rational knots, in Proceedings of the 37th Taniguchi symposium on topology and teichmuller spaces held in Finland, July 1995 (Kojima, S., Matsumoto, Y., Saito, K. and Seppälä, M., Editors) (1996), 31-35.

12. C. Hodgson, Degeneration and regeneration of hyperbolic structures on threemanifolds, Thesis (Princeton, 1986).

13. C. Hodgson and S. Kerckhoff, Rigidity of hyperbolic cone-manifolds and hyperbolic Dehn surgery, J. Diff. Geom. 48(1) (1998), 1-59.

14. H. Hopf, Über die Abbildungen der dreidimensionalen Sphäre auf die Kugelfläche, Math. Ann. 104 (1931), 637-665.

15. I. Izmestiev, Examples of infinitesimally flexible 3-dimensional hyperbolic conemanifolds, J. Math. Soc. Japan 63(2) (2011), 581-598.

16. S. Kojima, Deformations of hyperbolic 3-cone-manifolds, J. Diff. Geom. 49(3) (1998), 469-516.

17. A. A. Kolpakov and A. D. Mednykh, Spherical structures on torus knots and links, Siberian Math. J. 50(5) (2009), 856-866.

18. G. Montcouquiol, Deformation of hyperbolic convex polyhedra and 3-cone-manifolds, Geom. Dedicata (2012), arXiv:0903.4743.

19. G. D. Mostow, Quasi-conformal mappings in n-space and the rigidity of hyperbolic space forms, Inst. Hautes Etudes Sci. Publ. Math. 34 (1968), 53-104.

20. J. Porti, Regenerating hyperbolic and spherical cone structures from Euclidean ones, Topology 37(2) (1998), 365-392. 852.

21. J. Porti, Regenerating hyperbolic cone structures from Nil, Geom. Topol. 6 (2002), 815

22. J. Porti, Spherical cone structures on 2-bridge knots and links, Kobe J. Math. 21(1-2) (2004), 61-70.

23. J. Porti, Regenerating hyperbolic cone 3-manifolds from dimension 2, Ann. Inst. Fourier, arXiv:1003.2494.

24. G. Prasad, Strong rigidity of Q-rank 1 lattices, Invent. Math. 21 (1973), 255-286.

25. J. Ratcliffe, Foundations of hyperbolic manifolds (Springer-Verlag, New York, 1994). (Graduate Texts in Math.; 149).

26. G. de Rham, Reidemeister's torsion invariant and rotations of $S^{n}$, Differential analysis, Bombay Colloq. (Oxford University Press, London, 1964), 27-36.

27. M. Rothenberg, Torsion invariants and finite transformation groups, Proc. Symposia Pure Math. 32 (1978), 267-311.

28. J.-M. Schlenker, Dihedral angles of convex polyhedra, Discrete Comput. Geom. 23 (2000), 409-417.

29. W. P. Thurston, Geometry and topology of three-manifolds (Princeton University, 1979). (Princeton University Lecture Notes) 
30. E. B. Vinberg, Editor, Geometry II. Spaces of constant curvature (Springer-Verlag, New York, 1993). (Encyclopaedia of Mathematical Sciences; 29)

31. H. Weiß, Local rigidity of 3-dimensional cone-manifolds, J. Diff. Geom. 71(3) (2005), 437-506.

32. H. Weiß, Global rigidity of 3-dimensional cone-manifolds, J. Diff. Geom. 76(3) (2007), 495-523.

33. H. Weiß, The deformation theory of hyperbolic cone-3-manifolds with cone-angles less than $2 \pi$, Geom. Topol., arXiv:0904.4568. 